\title{
Left and right hemisphere involvement in speech perception: Electrophysiological correlates
}

\author{
DENNIS L. MOLFESE \\ Southern Illinois University, Carbondale, Illinois 62901
}

\begin{abstract}
Auditory evoked potentials (AEPs) were recorded from the left and right hemispheres of 10 adults who were listening to a series of auditory stimuli which varied along linguistic and acoustic dimensions. Discrete components of the brain's electrical response to these different stimuli were isolated and identified. Phonetic distinctions based on transitional elements occurred only in the left hemisphere. Other cortical components were identified which were sensitive to hemisphere differences, place of articulation cues, and formant bandwidth. One component of the brain's activity reflected hand preferences independently of hemispheric differences.
\end{abstract}

In recent years, researchers investigating speech perception have succeeded in identifying a number of components of the speech signal which provide the language listener with important cues necessary for language comprehension (Liberman, Cooper, Shankweiler, \& Studdert-Kennedy, 1967). These cues include factors such as formant frequency (Delattre, Liberman, Cooper, \& Gerstman, 1952), formant structure (Cutting, 1974), voice onset time (Lisker \& Abramson, 1964), and formant transitions (Cutting, 1974; Delattre, Liberman, \& Cooper, 1955; Liberman, 1957). Several of these cues have been found to be processed by different cortical regions (Cutting, 1974; Molfese, in press; Shankweiler \& Studdert-Kennedy, 1967; Studdert-Kennedy \& Shankweiler, 1970). Cutting, using a modified dichotic listening procedure, presented individuals with a series of computer-synthesized consonant-vowel (CV) and vowel (V) syllables. These syllables consisted of either normal formant (NF) structure with bandwidths of 60,90 , and $120 \mathrm{~Hz}$ for Formants 1, 2, and 3, respectively, or syllables with sine-wave formants (SF) characterized by bandwidths of $1 \mathrm{~Hz}$. The $\mathrm{CV}_{\mathrm{NF}}$ and the $\mathrm{CV}_{\mathrm{SF}}$ syllables contained an initial frequency transition which identified a specific consonant, while the $\mathrm{V}_{\mathrm{NF}}$ and $\mathrm{V}_{\mathrm{SF}}$ syllables did not contain a consonant transition. Cutting found that both bandwidth and transition factors influenced discriminations. Stimuli with normal formant structure or which contained an initial transition element

The author acknowledges the assistance of Mr. Andrew Papanicoloau during the testing sessions. This research was supported in part by a grant (2-15-87) from the Office of Research and Projects, Southern Illinois University at Carbondale. The stimuli were developed and constructed by Dr. James Cutting at the Haskins Laboratories. His kindness in providing these stimuli is gratefully acknowledged. Requests for reprints should be addressed to Dennis L. Molfese, Department of Psychology, Southern Illinois University, Carbondale, Illinois 62901. were better discriminated by the right ear (RE). Since the RE is known to have nearly $80 \%$ of its connections with the left hemisphere ( $\mathrm{LH})$, Cutting reasoned that these findings reflected different processing capabilities of the two hemispheres. Cutting further noted that perception of transitions occurred in the LH independent of formant structure. However, in a related study (Experiment 4), Cutting found that the LH apparently did not discriminate between phonetic and nonphonetic transitions-those that were linguistically meaningful vs. those that were not.

A recent study (Molfese, Nunez, Seibert, \& Ramanaiah, 1976) with newborn human infants failed to support Cutting's contention that only the $\mathrm{LH}$ processes transitional and formant structure information. Auditory evoked potentials (AEP) were recorded from left and right temporal scalp regions of 16 neonates within $48 \mathrm{~h}$ of birth in response to stimuli identical to those used by Cutting. The averaged AEPs were submitted to a principal components analysis. Four factors identified as accounting for $96 \%$ of the total variance were submitted to independent analyses of variance. These factors were found to be sensitive to sex and hemisphere differences as well as to changes in speech cues such as formant structure and transition. However, no interactions occurred between cortical components which were sensitive to changes in formant structure or transition and hemispheres. Both hemispheres apparently responded to the speech cues in a similar manner. Although these data did not support Cutting's findings, failure to find such interactions could have been due to maturational factors, differences in methodology, or task differences. There are obviously large maturational differences between the neonates of the Molfese et al. study and the adults in Cutting's work. Although general hemispheric differences may, in fact, be present at birth (Crowell, Jones, Kapuniai, \& Nakagawa, 1973; Davis \& Wada, 1977; Molfese, 1972; Molfese, 
Freeman, \& Palermo, 1975), more specific mechanisms within the left hemisphere could develop later as a function of maturation and language acquisition. Such differences could also indicate that AEP and dichotic procedures measure different mechanisms or that one technique is a more sensitive measure of neurolinguistic processes. Hemispheric activities responsible for the RE advantage may not be reflected in AEPs recorded from only one site over each hemisphere. It should be noted, however, that Cutting's findings were based on relatively small, albeit reliable, differences. For example, in Experiment 3 (Cutting, 1974) the mean group data indicate that the RE accounted for 69 of 96 correct responses in the CV condition while 55 of 96 correct responses characterized the LE trials. Fourteen correct responses separated the performance of the two ears. In the $\mathrm{V}$ condition, this difference was reduced to six trials, with the RE making 66 correct responses while the left ear accounted for 60 correct responses. Given the scoring procedures employed by Cutting, in which a correct identification resulted in a score of 2 correct responses while an incorrect one was scored as 2 incorrect responses, performance on only seven trials ( 7 correct identifications) separated ears in the $\mathrm{CV}$ condition while only three trials were responsible for the RE advantage in the $V$ condition. Although such small differences in performance were found to be reliable, Cutting overlooked the fact that LE performance, though lower than RE performance, was still well above chance levels. This observation, when viewed in light of the small differences in scores which separated the performance of the two ears, suggests that the two ears may be drawing on somewhat comparable mechanisms during this task. Freides (1977) recently argued that techniques such as dichotic listening procedures which employ competition and masking measures are heavily influenced by output factors. Cutting's procedures, then, may have measured response strategies rather than processing dominance. The failure of Molfese et al. to replicate Cutting's RE effect with AEP procedures may have been due to the fact that AEPs were more sensitive measures of hemispheric processing of incoming information.

In order to appropriately evaluate Cutting's findings with the electrophysiological procedures, these materials should be presented to an adult population. As noted above, differences in the findings reported by Cutting (1974) and Molfese et al. (1976) may have been due to differences in the maturational levels of the two populations. In the present study, stimuli similar to those employed by Cutting were presented to adults to determine if AEP procedures can be used to identify speech cues which might be differentially processed by the two hemispheres. These included one set of stimuli with phonetic transitions which were recognized as specific consonant sounds while a second set contained nonphonetic transitions. These stimuli were recognized as "speech-like" but could not be identified. Differences obtained in responses to these two sets of stimuli might suggest the presence of linguistic feature detectors (Cutting, 1974). In addition, any evidence of differential hemispheric responding to these stimuli would serve to further localize these mechanisms within one hemisphere. Although Cutting had failed in his earlier attempt to find such differences with his temporal order procedures, it was thought that techniques which directly assessed cortical activity and sensory input might be more sensitive to such differences than those that relied on output strategies (Freides, 1977).

In light of electrophysiological evidence suggesting some relation between differential cerebral activity and handedness (Provins \& Cunliffe, 1972; Raney, 1939), as well as clinical reports concerning an interaction between linguistic functions and handedness (Hecaen \& Ajuriaguerra, 1964; Zangwill, 1960), attempts were made to assess possible relationships between handedness and hemispheric factors in speech perception.

\section{METHOD}

\section{Subjects}

Two male and eight female adults, ranging in age from 18.1 years to 22.0 years (mean age, 19.5 years), participated in this experiment. The Edinburgh Inventory for Handedness (Oldfield, 1971) was administered to all participants to determine hand preferences. The overall average group Laterality Quotient (LQ) was 74.4 , with a range of 25.0 to 100.0 . These participants were then divided into two groups of five subjects, a high right-hand preference group (mean LQ $=93.8$, with a range of 82 to 100 ) and a low right-hand preference group (mean LQ $=52.4$, with a range of 25 to 78 ).

\section{Stimuli}

Eight consonant-vowel syllables constructed on the Haskins parallel resonance synthesizer were employed. All stimuli consisted of an initial rapid-frequency transition, $50 \mathrm{msec}$ in length, followed by steady-state formant elements, $300 \mathrm{msec}$ in duration. Rise times were identical for all stimuli. The eight stimulus items represented four different stimulus sets. The first two sets consisted of two $\mathrm{CV}_{\mathrm{NF}}$ and two $\mathrm{CV}_{\mathrm{SF}}$ stimuli corresponding to the consonant vowel syllables /bae, gae/. The $\mathrm{CV}_{\mathrm{NF}}$ stimuli were characterized by normal formant structure with bandwidths of 60,90 , and $120 \mathrm{~Hz}$ for formants 1,2 , and 3 , respectively. The formants of the $\mathrm{CV}_{\mathrm{SF}}$ stimuli consisted of $1-\mathrm{Hz}$ sine waves whose frequencies matched the middle values of the $\mathrm{CV}_{\mathrm{NF}}$ formants. Two other sets of stimuli consisted of two $\mathrm{CV}$ stimuli $\left(\mathrm{C}^{\prime} \mathrm{V}_{\mathrm{NF}}\right)$ similar in structure to the $\mathrm{CV}_{\mathrm{NF}}$ stimuli, with the exception that the formant transitions could not have been produced by a human vocal tract, and two $\mathrm{CV}$ stimuli $\left(\mathrm{C}^{\prime} \mathrm{V}_{\mathrm{SF}}\right.$ ) similar to the $\mathrm{CV}_{\mathrm{SF}}$ stimuli, with the exception that their transitions were identical to the $C^{\prime} V_{N F}$ stimuli. An example of each stimulus type for the /bae/ syllable is presented in Figure 1. The use of $\mathrm{C}^{\prime} \mathrm{V}$ and $\mathrm{CV}$ stimuli would provide an opportunity to assess the contributions of phonetic (CV) and nonphonetic $\left(\mathrm{C}^{\prime} \mathrm{V}\right)$ transitions to differential hemispheric activity. For additional information concerning the nature of these stimuli, 


\section{PHONETIC TRANSITION}

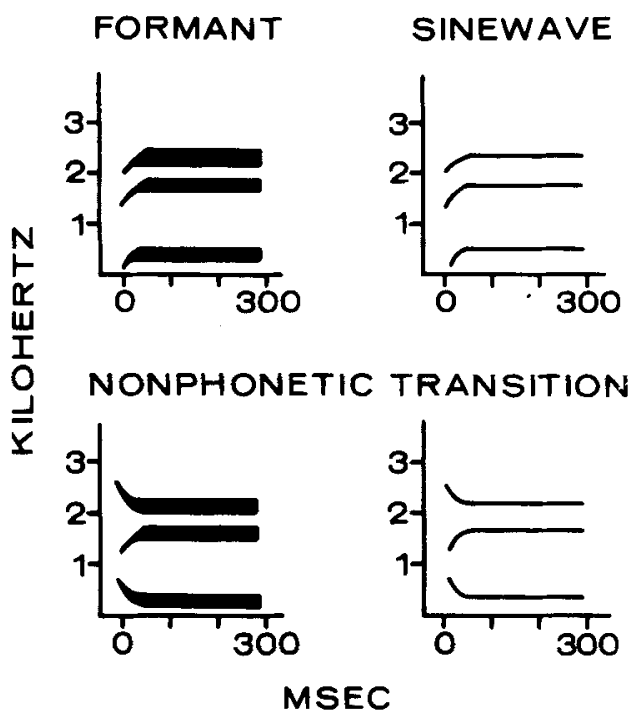

Figure 1. Schematic spectrograms of the speech (normal formant structure) and nonspeech (sine-wave formant structure) stimuli with phonetic $(C V)$ and nonphonetic $\left(C^{\prime} V\right)$ transitions (after Cutting, 1974).

the reader is referred to Cutting (1974). Sixteen different orderings of the eight stimuli were recorded on one channel of a stereo tape recorder (Sony Model TC-560). The time interval between stimuli was randomly varied from 4 to $8 \mathrm{sec}$ in order to reduce the effects of expectation and habituation. A $1 / 4-\mathrm{V} 50-\mathrm{Hz}$ squarewave pulse, time-locked to the onset of the stimulus, was recorded on a second channel of the tape. This pulse later served to identify the beginning of each stimulus presentation and the auditory evoked potential for a PDP- 12 computer.

\section{Procedure}

Subjects were tested individually while seated in a reclining chair in a sound-dampened and electrially shielded chamber. The stimuli were presented through a speaker centered and suspended approximately $1 \mathrm{~m}$ directly above the individual's head. The stimulus peak intensity at the subject's ears was $80 \mathrm{~dB}$ SPL. Grass silver electrodes were placed on the scalp over the superior temporal regions of the left and right hemispheres at $T_{3}$ and $T_{4}$ of the 10-20 Electrode System of the International Federation (Jasper, 1958) and referred to linked ear lobes. Electrode impedances for each side of the head were checked and recorded before and after the testing session for each subject. The mean values of these resistances were $2.8 \mathrm{~K} \Omega$ before testing (range $=1.0-4.8$ ) and $3.3 \mathrm{~K} \Omega$ (range $=1.4-5.8$ ) at the end of the 15-min testing session. Resistances over the left and right leads were maintained within $.5 \mathrm{kHz}$ of each other. The electrodes were connected to two isolation amplifiers (Analogue Devices, Model 273J). This output, in turn, was connected to two modified differential amplifiers (Tektronix AM 502), with the bandpass flat between .1 and $30 \mathrm{~Hz}$ and with gain settings at $20 \mathrm{~K}$. AEPs elicited in response to the stimuli were recorded on a Vetter modified cassette FM tape recorder (Model C-4) for later analyses.

\section{RESULTS}

The AEPs from each subject were digitized and averaged on a PDP -12 computer using a modified version of "Averager" (Decus No. 12-84). Averages were based on 16 repetitions for each stimulus. The group-averaged AEPs for the two hemispheres in response to the eight different stimulus conditions are presented in Figure 2.

Analyses were performed on the AEPs using factoranalysis and analysis of variance techniques (Chapnian, McCrary, Bragdon, \& Chapman, 1978; Molfese et al., 1976). The data set consisted of the digitized amplitude values of 160 averaged evoked potentials which were obtained from the two hemispheres of 10 adults for eight different stimuli. An input data matrix was obtained from the 160 averaged AEPs by taking the amplitude values at each of the 100 time points at intervals of $5 \mathrm{msec}$ over the $500 \mathrm{msec}$ period following stimulus onset. These data were then submitted to a principal components analysis (BMD08M) using the BMDX72 program package (Dixon, 1972). This program first transformed the data matrix into a correlation matrix. The principal components analysis was then applied to a 100 by 100 matrix which consisted of the product moment correlations computed for each pair of time points. Factors which met the eigen value $=1.0$ criterion were retained. This criterion insured that only factors which accounted for at least as much variance in the data as any one of the original 100 variables would be retained. In this manner, six factors accounting for $97.02 \%$ of the total variance were isolated. These were then rotated using the normalized varimax criterion which preserved the orthogonality among the factors while improving their distinctiveness. Factor scores (gain factors) were then computed for each of the 160 original AEPs for each of the six rotated principal components.

The centroid and the six factors generated by the principal components analysis are plotted in Figure 3. The centroid is the average evoked potential for all subjects in all conditions and consequently reflects the electrocortical activity common to all the AEPs. The six factors each consists of 100 factor loadings which correspond to the 100 time points. These factor loadings reflect the association of the factors to the original variables (time points). The waveform for Factor 1 was characterized by an early peak at $70 \mathrm{msec}$ and a second peak $300 \mathrm{msec}$ after stimulus onset. Factor 2 was marked by a relatively late peak $455 \mathrm{msec}$ after stimulus onset. Factor 3 remained flat until midway through the AEP response when a wave peaked at approximately $305 \mathrm{msec}$. Factor 4 was marked by a major peak at $180 \mathrm{msec}$, while Factor 5 was characterized by major peaks at 135,240 , and $350 \mathrm{msec}$ following stimulus onset. A peak at $250 \mathrm{msec}$ and a second at $380 \mathrm{msec}$ characterized Factor 6.

Independent analyses of variance of Handedness (2) by Formant Structure (2) by Consonant (2) by 


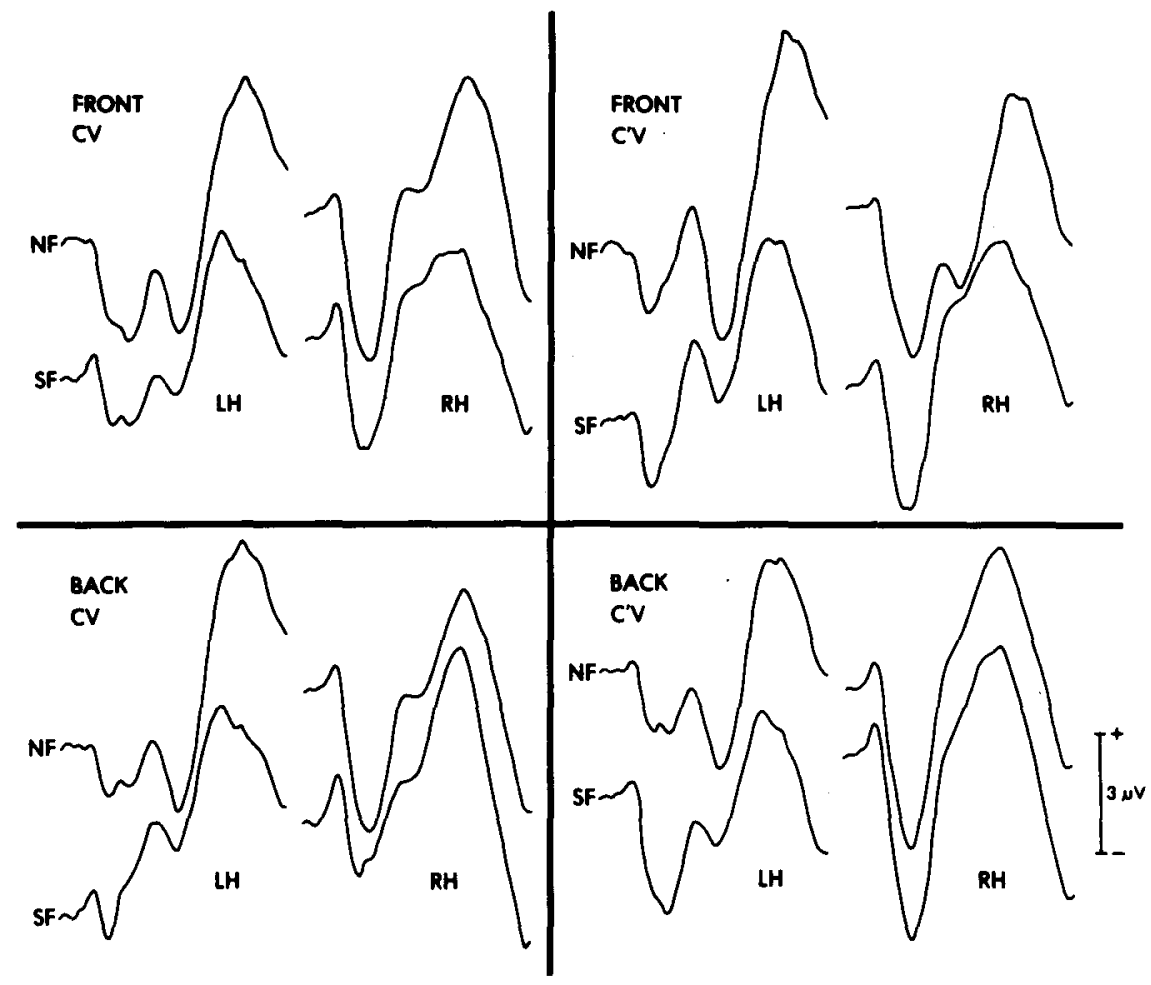

Figure 2. The group average AERs recorded from the two hemispheres in response to all stimulus conditions. AEP duration is $\mathbf{5 0 0}$ msec.

Transition (2) by Hemisphere (2) were performed on the factor scores for each factor. A Consonant by Transition by Hemisphere interaction $(F=27.72, \mathrm{df}=$ $1,8, p<.001)$ characterized Factor 1 . This interaction is presented in Figure 4. Post hoc Scheffé tests of this interaction indicated that the $\mathrm{LH}$ responses for the front and for the back consonants differentiated between stimuli with phonetic and nonphonetic transitions. No differences were found in the RH for these sounds. The group-averaged AEPs for the left and right hemispheres for the $\mathrm{CV}$ and $\mathrm{C}^{\prime} \mathrm{V}$ stimuli are illustrated in Figure 5. The amplitude of the P80 component for the LH CV condition is less than that for the P240 component. The amplitudes are the same for both components in the $C^{\prime} \mathrm{V}$ condition. For both RH AEPs, the amplitude of the P240 component is less than that for the P80 component. Significant main effects for Formant Structure $(F=23.87$, df $=1,8, p<.001)$ and Hemispheres $(F=18.46$, df $=1,8, p<.01)$ characterized Factor 2. Significant main effects for Formant Structure $(\mathrm{F}=43.09, \mathrm{df}=1,8, \mathrm{p}<.0001)$ and Hemispheres $(F=37.75, \mathrm{df}=1,8, \mathrm{p}<.001)$ were found for Factor 3. The group-averaged AEPs for the normal and sine-wave formant stimuli are presented in Figure 6. The $\mathrm{N}_{2}$ component is more marked for the $\mathrm{NF}$ than in the SF stimuli. A marginal effect for Handedness $(F=7.36, \mathrm{df}=1,8, \mathrm{p}<.03)$ characterized Factor 5, while a Handedness by Consonant interaction $(F=10.20$, df $=1,8, p<.015)$ was found for Factor 6. This interaction reflected a difference in responding to $/ \mathrm{b} /$ by individuals with high and low right-hand preferences.

\section{DISCUSSION}

A number of factors underlying the AEP were found to reflect differences in degree of righthandedness, hemispheres, phonetic transitions, formant structure, and consonants.

Several factors reflected differences in formant structure and hemispheres independent of other experimental variables (Factors 2 and 3 ). These findings are in agreement with earlier AEP studies with infants (Molfese et al., 1976) and differ from those reported by Cutting (1974). Although maturational and experiential differences in populations as possible sources of this discrepancy were eliminated 

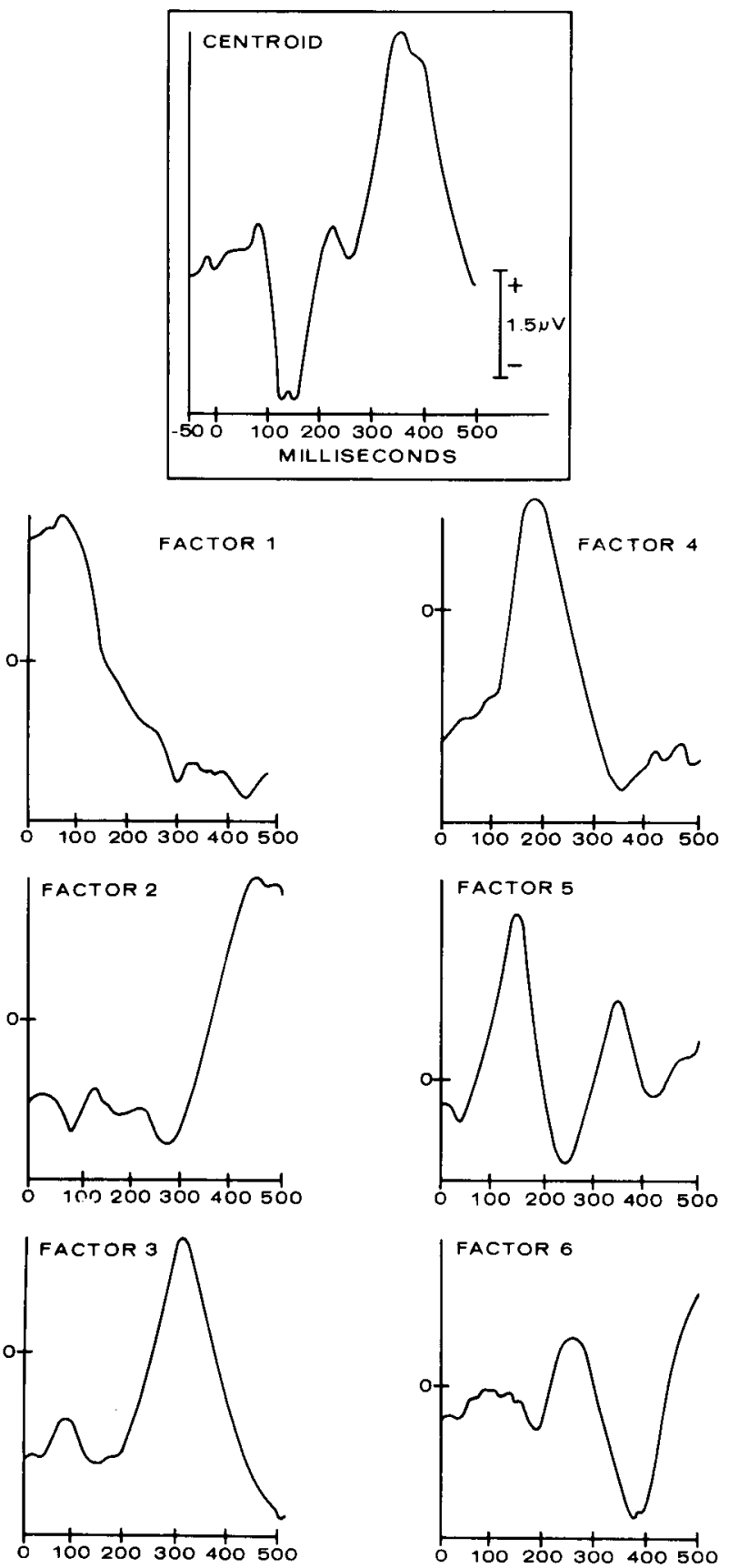

Figure 3. The centroid (the average AEP for the entire data set) and the six factors obtained from the varimaxed principal components analysis. The time course is $\mathbf{5 0 0}$ msec with stimulus onset at 0 msec. The calibration scale for the centroid is $1.5 \mu \mathrm{V}$, with positive up. The vertical scale for the factor loadings are arbitrary. The factor scores or gain factors (the dependent variable in the analyses of variance) for the different conditions determine the polarity and amplitude of these factors. Plots were based on points at 10 -msec intervals.

in the present study with the testing of adult subjects, the failure to replicate Cutting's hemisphere effect for bandwidth could be due to several factors, as noted earlier. The electrophysiological measures employed in the present study may not be sensitive to the factors responsible for the ear differences reported by Cutting. The electrodes may not have been near cortical regions where such activity occurs. AEPs were recorded from only one scalp location over each hemisphere. Perhaps if more sites were employed, hemisphere differences related to formant structure might be found. A second possibility also exists. This involves the discussion developed earlier concerning the possible confounding of hemisphere effects in Cutting's study with response strategies. The AEPs might actually be more sensitive to the cortical processing of incoming information and less sensitive in the present case to output strategies. Clearly, more work is needed to resolve this issue.

A factor (Factor 1) that reflected specific cortical responses to transition elements was also identified. Although the initial portion of this factor occurred

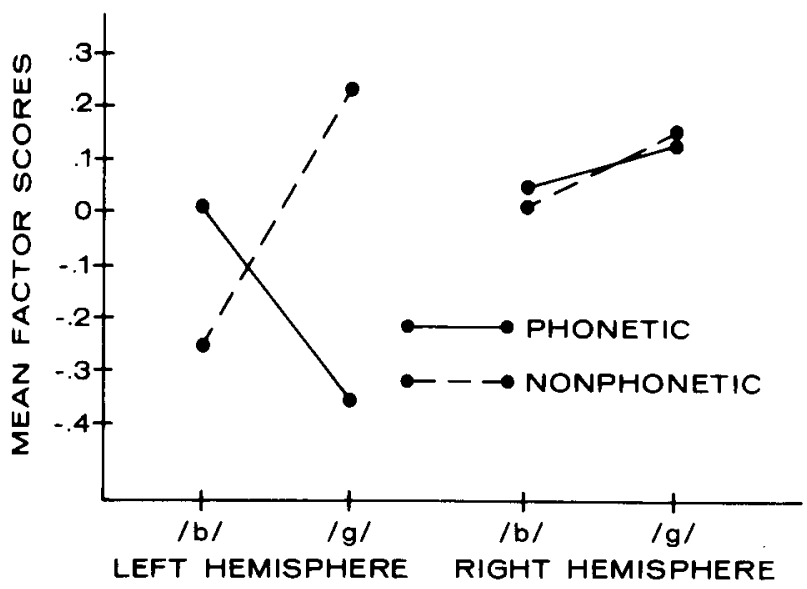

Figure 4. Mean factor scores for Factor 1 for each hemisphere in response to the $/ \mathrm{b} /$ and $/ \mathrm{g} /$ stimuli which varied in phonetic transition. These factor scores indicate the amount of each factor that is present in the AEP for a particular condition.

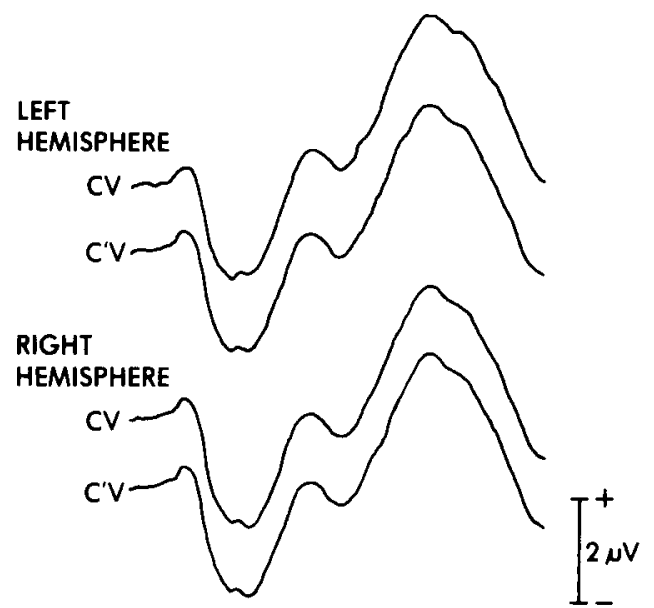

Figure 5. The group-averaged left and right hemisphere AEPs for the $C V$ and $C^{\prime} V$ stimuli irrespective of formant structure. The calibration marker is $2 \mu$ with positive up. Latency is 500 msec. 


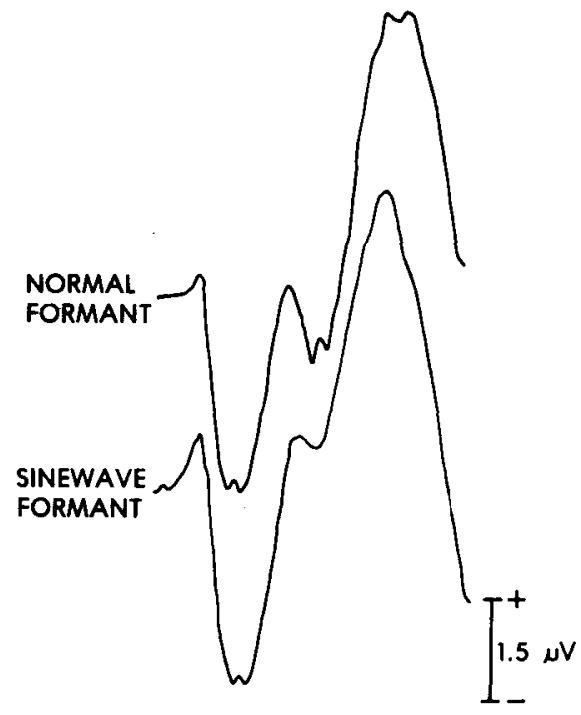

Figure 6. The group-averaged AEPs for the normal and sinewave formant stimuli. The calibration marker is $1.5 \mu \mathrm{V}$ with positive up.

during the period of the consonant transition, a later component of this factor overlapped in time the vowel portion of the CV syllable. Analyses indicated that only the $\mathrm{LH}$ differentiated between phonetic transitions for the $/ \mathrm{b} /$ and $/ \mathrm{g} / \mathrm{stimuli}$. These stimuli did differ phonetically. That is, the CV stimuli contained transitions which characterized specific American English language speech sounds, while the C'V stimuli did not. Such findings, although hypothesized by Cutting, were not found in his earlier research. This effect could well indicate that transitional cues may be processed by phonetic mechanisms within the LH.

General hemispheric differences were found (Factors 2 and 3) which did not interact with other variables. Such findings of differential hemispheric activity independent of stimulus or task variables only indicate that the two hemispheres, as reflected in these factors, were responding differently to the stimuli. Both hemispheres were actively responding to all stimuli. Neither factor indicates that one hemisphere was "more"' involved in processing the material than the other (Molfese et al., 1976).

As noted earlier, the relation of hand preferences to hemispheric processes has long been a topic of interest to individuals investigating brain-language functions. It has generally been believed that some relationship exists between differential hemispheric processing of language information and hand preferences. However, no such relationships were found in the present study. One component (Factor 5) reflected degree of right-hand preferences across all subjects and stimulus variables. Where interactions of handedness and experimental variables did occur, the effects were similar for both hemispheres. The absence of a Hemisphere by Handedness interaction fails to support the notion that there is a strong, positive relationship between hemispheric differences and degree of right-handedness. Further studies with both dextrals and sinistrals are needed before this question can be resolved. There was, however, some indication that degree of handedness was related to processing of the stimulus information (Factor 6). For example, different cortical components characterized $/ b /$ for the high and low handedness groups. It is not clear how to interpret such findings. Nevertheless, since handedness did relate to some aspects of speech perception and, perhaps to some more general cortical difference, future research into human electrocortical processes should control for this variable.

\section{SUMMARY}

Auditory evoked potentials were recorded from adults in response to a series of materials which varied along a number of linguistic and acoustic dimensions. Portions of the brain's electrical response to these different stimuli were isolated and identified. Phonetic distinctions based on transitional elements appeared to occur primarily in the left hemisphere. A component of the cortical response was also found which reflected differences in degree of right-handedness.

\section{REFERENCES}

Chapman, R. M., MCCrary, J. W., Bragdon, H. R., \& Chapman, J. A. Latent components of evoked potentials functionally related to information processing. In J. E. Desmedt (Ed.), Progress in clinical neurophysiology (Vol. 6) Cognitive components in cerebral event-related potentials and selective attention. Basel: Karger, 1978.

Crowell, D. H., Jones, R. H., Kapuniai, L. E., \& Nakagawa, J. K. Unilateral cortical activity in newborn humans: An early index of cerebral dominance. Science, 1973, 180, 205-208.

Curting, J. E. Two left-hemisphere mechanisms in speech perception. Perception \& Psychophysics, 1974, 16, 601-612.

DAvis, A. E., \& WADA, J. A. Hemispheric asymmetries in human infants: Spectral analysis of flash and click evoked potentials. Brain and Language, 1977, 4, 23-31.

Delattre, P. C., Liberman, A. M., \& Cooper, F. S. Acoustic loci and transitional cues for consonants. Journal of the Acoustical Society of America, 1955, 27. 769-773.

Delattre, P. C., Liberman, A. M., Cooper, F. S., \& Gerstman, L. J. An experimental study of the acoustic determinants of vowel color: Observations on one and two formant vowels synthesized from spectrographic patterns. Word, 1952, 8, 195-210.

Dixon, W. J. (Ed.) BMD biomedical computer program: $X$-series supplement. Berkeley: University of California Press, 1972.

Freides, D. Do dichotic listening procedures measure lateralization of information processing or retrieval strategy. Perception \& Psychophysics, 1977, 21, 259-263.

Hecaen, H.. \& AJuriaguerra, J. de Left handedness. New York: Grune \& Stratton, 1964. 
JASPER, H. H. The ten-twenty electrode system of the international federation of societies for electroencephalography: Appendix to report of the committee on methods of clinical examination in electroencephalography. Journal of Electroencephalography and Clinical Neurophysiology, 1958, 10, 371.

Liberman, A. M. Some results of research on speech perception. Journal of the Acoustical Society of America, 1957, 29, 117-123.

Liberman, A. M., Cooper, F. S., Shankweiler, D., \& Studdert-Kennedy, M. Perception of the speech code. Psychological Review, 1967, 74, 431.461.

LiSkER, L., \& ABramson, A. S. Stop categories and voice onset time. Proceedings of the Fifth International Congress of Phonetic Sciences, Muster, Hungary, August 1964.

Molfese, D. L. Cerebral asymmetry in infants, children and adults: Auditory evoked responses to speech and music stimuli. Unpublished doctoral dissertation, Pennsylvania State University, 1972.

Molfese, D. L. Neuroelectrical correlates of categorical speech perception in adults. Brain and Language, 1978, 5, 25-35.

Molfese, D. L., Freeman, R. B., \& Palermo, D. S. The ontogeny of brain lateralization for speech and nonspeech stimuli. Brain and Language, 1975, 2, 356-368.
Molfese, D. L., Nunez, V., Seibert, S. M., \& Ramanaiah, N. V. Cerebral asymmetry: Changes in factors affecting its development. Annals of the New York Academy of Sciences, 1976, 280, 821-833.

Oldfield, R. C. The assessment and analysis of handedness:

The Edinburgh Inventory. Neuropsychologia, 1971, 9, 97-113.

Provins, K. A., \& Cunliffe, P. The relationship between EEG activity and handedness. Cortex, 1972, 8, 136-146.

RANEY, E. T. Brain potentials and lateral dominance in identical twins. Joumal of Experimental Psychology, 1939, 24, 21-39.

SHANKWEILER, D., \& STUDDERT-KENNEDY, M. Identification of consonants and vowels presented to left and right ears. Quarterly Journal of Experimental Psychology, 1967, 19, 59-63.

Studdert-Kennedy, M., \& ShankWeiler, D. Hemispheric specialization for speech perception. Journal of the Acoustical Society of America, 1970, 48, 579-594.

ZANGWILL, O. L. Cerebral dominance and its relation to psychological function. Edinburgh: Oliver \& Boyd, 1960.

(Received for publication October 20, 1977; revision accepted December 22, 1977.) 